\title{
Reentrant behaviour and universality in the Anderson transition
}

\author{
S. L. A. de Queiroz* \\ Instituto de Física, Universidade Federal do Rio de Janeiro, \\ Caixa Postal 68528, 21945-970 Rio de Janeiro RJ, Brazil
}

(November 2, 2018)

\begin{abstract}
The three-dimensional Anderson model with a rectangular distribution of site disorder displays two distinct localization-delocalization transitions, against varying disorder intensity, for a relatively narrow range of Fermi energies. Such transitions are studied through the calculation of localization lengths of quasi- one-dimensional systems by transfer-matrix methods, and their analysis by finitesize scaling techniques. For the transition at higher disorder we find the localization-length exponent $\nu=1.60(5)$ and the limiting scaled localization-length amplitude $\Lambda_{0}=0.57(1)$, strongly suggesting universality with the transition at the band centre, for which currently accepted values are $\nu=$ $1.57(2)$ and $\Lambda_{0}=0.576(2)$. For the lower (reentrant) transition, we estimate $\nu=1.55(15)$ and $\Lambda_{0}=0.55(5)$, still compatible with universality but much less precise, partly owing to significant finite-size corrections.
\end{abstract}

\section{INTRODUCTION}

According to Anderson's original scenariot, if one starts from a translationally invariant model Hamiltonian for noninteracting electrons and considers increasing quenched disorder (e.g. by assigning random self-energies to lattice sites), a critical threshold is reached at which quantum interference inhibits propagation, and the system undergoes a localization transition, from a diffusive (metallic) phase to an insulating (localized) regime. For the past twenty years, the framework for studies of critical properties of the Anderson transition has mgstly been provided by the scaling theory of localization $\mathrm{A}_{\mathrm{B}}$, Numerical studies have been progressively refined 13 , so that accurate estimates exist for several quantities of interest.

In the site-disordered version which will concern us here, the problem is usually formulated in terms of a tight-binding Hamiltonian on a regular lattice,

$$
\mathcal{H}=\sum_{i} \varepsilon_{i}|i\rangle\left\langle i\left|+V \sum_{\langle i, j\rangle}\right| i\right\rangle\langle j|
$$

where the site self-energies $\varepsilon_{i}$ are independent, identically distributed random variables obeying a specified distribution, $\langle i, j\rangle$ denotes nearest neighbours and the energy scale is set by the hopping matrix element, $V \equiv 1$. The intensity of disorder is generically represented by a width, $W$, of the self-energy probability distribution.

On a simple cubic lattice, for the non-random case (all $\varepsilon_{i}=\varepsilon_{0} \equiv 0$, for convenience) the Schrödinger equation $\mathcal{H}|\psi\rangle=E|\psi\rangle$ yields Bloch (propagating) states for $-6 \leq E \leq 6$ and no solutions outside this eperoy range. In many studies of the localization transition 16010 one looks for destruction of transmission at the band centre, that is, only the behaviour of states at $E=0$ is considered. Since that is as far as possible from the unperturbed band edges, one is maximising the direct influence of quenched disorder which, coupled with the ever-present quantum interference effects, induces Anderson localization (compare this with the purely quantum mechanism responsible for the very existence of a band of allowed states, and forbidden ones outside it, even in a homogeneous system).

Further studies have investigated the $E \neq 0$ region, attempting to map the mobility edge trajectory in the $E-W$ pland 914 . Along this latter line, reentrant behaviour was found for certain distributions of site selfenergies, in a nargon-range of energies above the unperturbed band edget 14: at fixed $E$, as $W$ decreases from some large initial value the system first displays an upper transition from insulator to metal at $W \rightarrow W_{u}^{+}$, much the same as at $E=0$; then, upon further reduction of disorder a second, lower transition takes place, back towards an insulating phase as $W \rightarrow W_{l}^{+}$. The mechanisms underlying each of these transitions arequalitatively understood, and fundamentally distinct the onset of extended states as $W \rightarrow W_{u}^{+}$is due to diminishing quantum interference, at $W \rightarrow W_{l}^{-}$it is the increasing overlap of (and consequent tunnelling between) localized states that eventually produces delocalized behaviour. Reentrant regions were found for rectangular $\left(P\left(\varepsilon_{i}\right)=\right.$ constant, $\left.\left|\varepsilon_{i}\right| \leq W / 2\right)$ and, to a lesser extent, for Gaussian $\left(P\left(\varepsilon_{i}\right) \propto \exp \left(-\varepsilon_{i}^{2} / 2 W^{2}\right)\right)$ distributions, and not at all for a Lorentzian one $\left(P\left(\varepsilon_{i}\right) \propto W /\left[\varepsilon_{i}^{2}+W^{2}\right]\right)$. This is consistent with the idea that, in order for localized states to coalesce into one extended wavefunction (the mechanism underlying the lower transition), their respective energies must not differ by much, thus enabling their mutual resonance. The narrower the distribution of self-energies (preferably with a sharp cutoff, as in the rectangular case), such situation is more likely to be found. For a Lorentzian shape, where already the second moment diverges, chances of overlap are negligible.

Though significant advances have been made recently regarding properties at the band centre 2013 , such as uni- 
versality (with respect to varying disorder distributions), corrections to scaling and accurate numerical estimates of critical quantities, a similar quantitative understanding of behaviour elsewhere on the phase diagram appears to be lacking. Here we set the model parameters in order to enable a study of the reentrant region, and probe both upper and lower transitions; our main goal is to find what universality class(es) they belong to. Our numerical procedures and some concurring technical aspects are discussed in Section III; the upper (high disorder) and lower (reentrant) transitions are analyzed respectively in Sections III and IV; conclusions and final remarks are given in Section $\mathrm{V}$.

\section{MODEL DETAILS AND CALCULATIONAL METHOD}

We consider the Hamiltonian given in Eq. (11), on a simple cubic lattice, with a rectangular distribution of site self-energies:

$$
P\left(\varepsilon_{i}\right)= \begin{cases}\text { constant } & -W / 2 \leq \varepsilon_{i} \leq+W / 2 \\ 0 & \text { otherwise }\end{cases}
$$

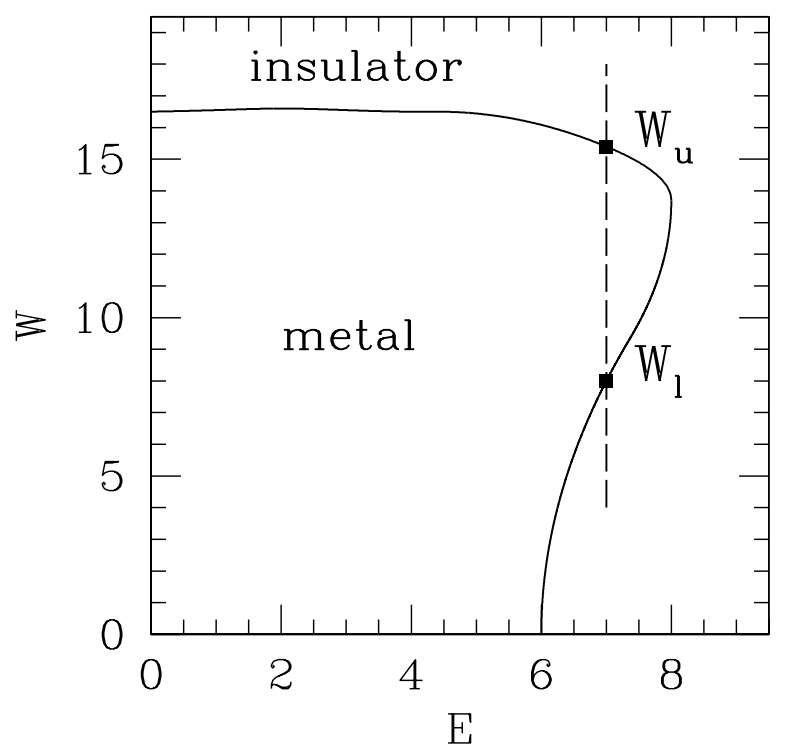

FIG. 1. Schematic phase diagram for rectangular disorder distribution (after Ref. 8, see their figure 1). Dashed line at $E=7$ shows region investigated here, for which the lower and upper transitions are located at $W_{l} \simeq 8, W_{u} \simeq 15$, respectively.

For such distribution the mobility edge trajectory, as found in Ref. 8, is semi-quantitatively depicted in Figure 1. From inspection of the Figure, we decided for fixing the energy at $E=7$ and scanning $W$. This way two well-separated transitions (respectively at $W_{u} \simeq 8$,
$\left.W_{l} \simeq 15\right)$ are expected to be present, so presumably one can focus on their individual properties one at a time.

We have considered quasi- one-dimensional systems, "bars" of cross-section $L \times L$ sites, with periodic boundary conditions across, and very long length $N$. We have used $L=4,6,8,10,12$ and 14; as explained below, the values of $N$ were determined by numerical convergence criteria, $10^{5}$ being typical.

Following well-known procedures introduced by Pichard and Sarmal (see also Refs. 6, 10 for detailed descriptions), we considered the transfer matrix connecting matrix elements of the Hamiltonian, Eq. (11), on consecutive cross-sections, and iterated it along the "infinite" direction, thus extracting the corresponding Lyapunov characteristic exponents. Of these, the most relevant here is the one with smallest modulus, which gives the largest localization length $\lambda_{L}(E, W)$ (slowest decay along the bar of transverse dimension $L$, at given $W$, for an electronic wave function with energy $E$ ).

According to finite-size scaling (FSS)15, the nondiverging quasi- one-dimensional lengths $\lambda_{L}$ scale linearly with $L$ (apart from corrections to scaling, to be discussed below) at the critical point of the threedimensional system. Therefore, plots of the scaled localization lengths $\Lambda_{L} \equiv \lambda_{L} / L$ (e.g. against $W$, for fixed $E)$ for different values of $L$ must cross, and they should do so twice if there are two distinct second-order transitions. Leaving aside, for the moment, the question of whether this is consistent with the single-parameter theory of localization (see Ref. 8), one is provided with a rough-and-ready criterion to locate the expected critical points.

In order to produce reliable numerical estimates from the conceptual framework described thus far, two points must be carefully considered. First, though the convergence of finite- $N$ estimates of Lyapunov exponents towards their asymptotic values is guaranteed in principle16.17, it may be hard to decide when it has taken place to a desired accuracy. As noted in Ref. 6, "for such very long strips the variation of $\lambda$ [as a function of $N$ ] from step to step is very small due to averaging, and this can lead to a false impression of convergence". Accordipgly, we have kept track of the variance among estimates 6 , in the following way. Each $10^{3}$ steps an estimate of $\lambda$ (taking into account all iterations from the origin up to the sampling point) would be produced; first, twenty consecutive estimates would be accumulated, and the average and RMS deviation among them calculated. At the end of each subsequent $10^{3}$ steps, a new average and deviation would be calculated, of the most recent twenty estimates. Therefore, in this moving average procedure, one keeps track of fluctuations along the latest 20,000 iterations. It was found that, in order to reach $0.1 \%$ fractional deviation one typically needs $80,000-130,000$ iterations (except for low disorder, $W \lesssim 6.5$, where similar figures are achieved within $\sim 50,000$ steps or fewer). Strictly speaking, this is no guaranteee that the corresponding final estimate will be accurate within $0.1 \%$, as a very 
slow drift may still be masked underneath apparently random fluctuations. In Ref. 11, $10^{6}-10^{7}$ iterations at the band centre are quoted as necessary in order to give $0.1 \%-0.05 \%$ accuracy, although the latter term is not assigned a specific operational definition; in the closely related Green's function calculations of Ref. 8, 1\% accuracy is claimed for $\sim 40,000-70,000$ steps. Having this in mind, it seems that our current definition of $0.1 \%$ relative fluctuation warrants perhaps $0.3 \%-0.4 \%$ accuracy for the localization length, at best. We used $0.1 \%$ relative fluctuations for all $L=4-10$ data, and for selected $L=12-14$ points; in the latter case, intermediate$W$ data were collected with $0.5 \%-1 \%$ fluctuations and checked for smooth variation against the more accurate ones. As shown below, this will not be the most important source of errors in the present case.

Secondly, in general there may be systematic distortions to the linear, single-scaling parameter picture, caused bynonlinearity of scaling fields and/or irrelevant variables18. A detailed study of such effects for the Anderson transition at the band centre has been undertaken recently 11 . The result was that such corrections are of sizable importance in the case: estimates of critical quantities produced when they are taken into account tend to be more consistently reliable and accurate than if they are disregarded. Following the notation of Ref. 11, near the critical disorder $W_{c}$, with $w \equiv\left(W_{c}-W\right) / W_{c}$, the scaled localization length $\Lambda_{L}$ must vary as:

$$
\Lambda_{L}=F\left(\chi L^{1 / \nu}, \psi L^{y}\right)
$$

where $\chi$ is the relevant variable, $\psi$ is the leading irrelevant variable, the exponent $\nu$ characterizes the divergence of the thermodinamic $(L \rightarrow \infty)$ localization length via $\lambda_{\infty} w^{-\nu}$, and $y<0$ is the leading irrelevant exponent 15.18. Since there is no transition for finite $L$, one assumes

$$
\Lambda_{L}=\sum_{n=0}^{n_{I}} \psi^{n} L^{n y} F_{n}\left(\chi L^{1 / \nu}\right)
$$

further, since (for finite $L$ ) $\chi$ and $\psi$ also are smooth functions of $w$, one expands both in power series as well; linearity is recovered if $\chi(w)=w($ as $\chi(0)=0$ must hold), $\psi(w)=\psi$ (constant), and only the lowest powers are kept $\left(n_{I}=1\right)$ in the Taylor series for $\Lambda_{L}$ :

$$
\Lambda_{L}=F_{0}\left(w L^{1 / \nu}\right)+\psi L^{y} F_{1}\left(w L^{1 / \nu}\right) .
$$

Nonlinearity (both in relevant and irrelevant variables) and higher-order corrections associated to the irrelevant variable may be incorporated by keeping higher-order terms in the respective expansions. This is done at the expense of having a larger number of parameters to fit, and must be accompanied by the corresponding quality of numerical data. Here, we found that the quality of our data was generally consistent with the linearized form, Eq. (5).

\section{UPPER TRANSITION}

In Figure 2 we display the raw data for scaled localization lengths near the upper transition, whose approximate location is estimated from Ref. 8 (see also Figure 1 above). Though data for each $L$ behave somewhat irregularly, there is an unmistakeable crossing of curves, roughly at the centre of the diagram.

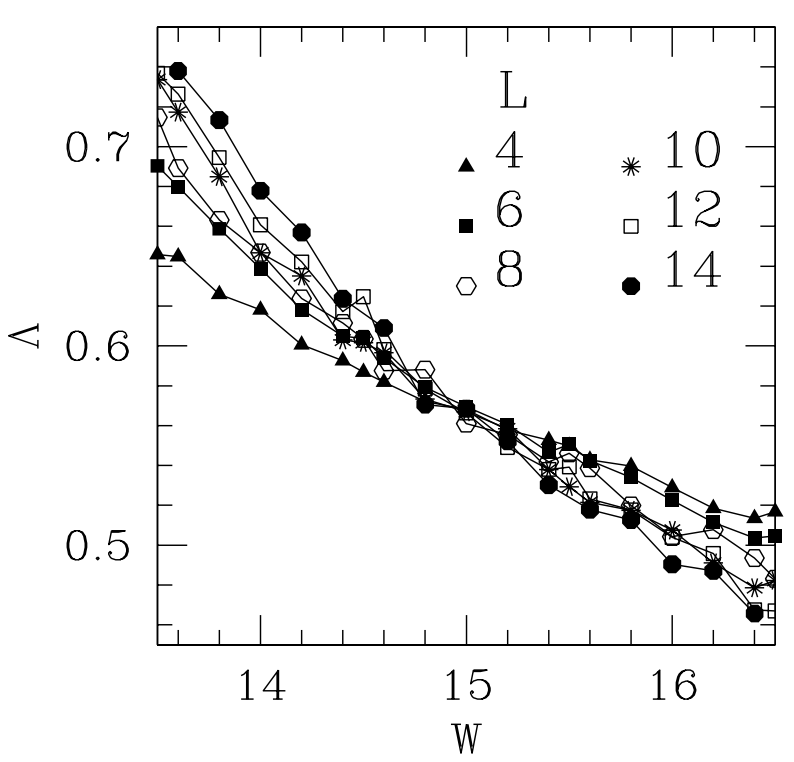

FIG. 2. Raw data for scaled localization lengths near the upper transition. Relative fluctuations, as defined in the text, are of order of symbol sizes or smaller.

Following along the lines proposed in Ref. 11, we attempted to fit assorted corrections to scaling. We found that, to be consistent with the range of variation and accuracy level of our data, the most sensible choice was (1) not to take nonlinearities of scaling variables into account, and (2) keep only up to quadratic terms in the Taylor expansion of $F_{1}\left(w L^{1 / \nu}\right)$ in Eq. (5) above. Thus we plotted

$$
\Lambda_{\text {corrected }} \equiv \Lambda_{L}-\psi L^{y}\left(1+a x+b x^{2}\right)
$$

against $x \equiv\left(W-W_{u}\right) L^{1 / \nu}$. This gave us six parameters to fit: $W_{u}, \nu, \psi, y, a$ and $b$. We started from reasonable guesses for some of these quantities (e.g. $W_{u} \simeq 15$, $|\psi|<1$ ); also, since this transition is driven by the same mechanism as that at the band centre, they might plausibly be in the same universality class, so $\nu \simeq 1.4-1.7$ seemed worth investigating (recall $\nu=1.57(2)$ from Ref. 11). With a few similar aditional assumptions, we checked the variation of $\chi^{2}$ against changes in the fitting parameters 19; the plot displayed in Figure 3 is representative of the best sort thus obtained. Though its (unweighted) $\chi^{2}=0.018$ for 110 data does not mean 
much by itself, it will be useful for comparison with the corresponding one at the lower transition, in Section IV. Corrections to scaling are of order up to $5 \%$ for $L=4$ and decrease steeply, owing to the large value of $|y|$ (similar to the picture at the band centre, see Table II of Ref. 11), to around $0.2 \%$ for $L=14$. The latter figure is close to the intrinsic numerical accuracy of raw data, so it is fair to say that corrections only play a significant role for the smaller sizes, say up to $L=8-10$. Our plots turned out to be quite sensitive against changes in $\nu$ of order $3-4 \%$ around the best-fitting value $\simeq 1.6$; therefore, the initial hypothesis of universality, as incorporated in the fitting procedure, appears to be rather consistent.

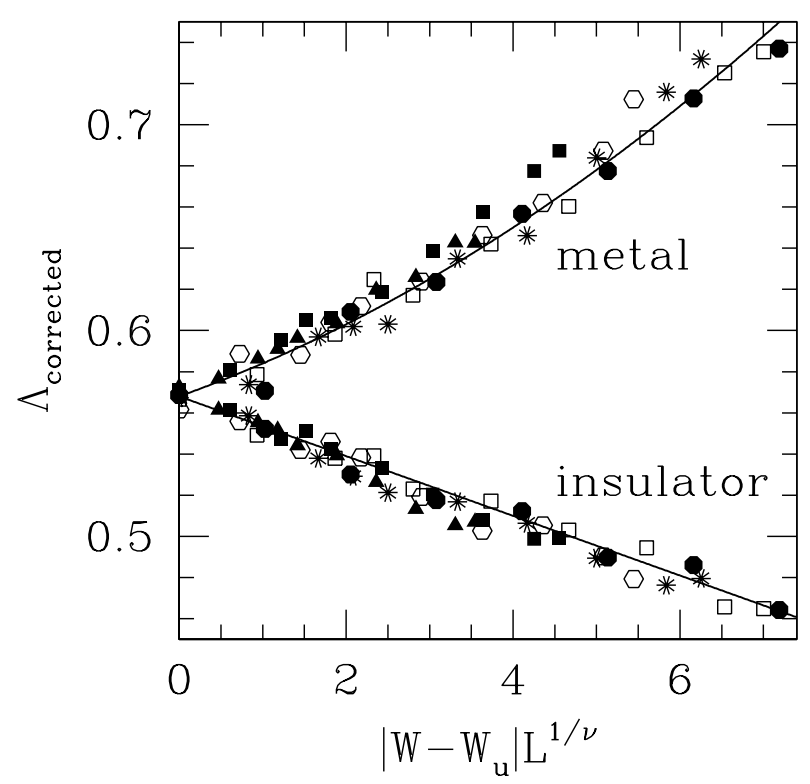

FIG. 3. Scaling plot near the upper transition. Here, $W_{u}=15.0, \nu=1.61, \psi=-0.21, y=-2.8, a=-0.21$, $b=-0.2$ (see Eq. (6)). Lines are guides to the eye, starting at $\Lambda_{0}=0.568$ on vertical axis. Key to symbols is the same as in Fig. 2.

On the other hand, the limiting amplitude, $\Lambda_{0} \equiv$ $\lim _{L \rightarrow \infty, W=W_{c}} \Lambda_{L}$, given on scaling plots such as Figure 3 by the intersection with the vertical axis, was found to be remarkably robust: we quote $\Lambda_{0}=0.57(1)$ for all reasonably behaved choices of parameters. This is in very good agreement with $\Lambda_{0}=0.576(2)$, a value found to be universal with respect to the form of disorder distribution at the band centre 11 .

TABLE I. Estimates of critical quantities at upper transition. Data for the band centre from Ref. 11. Comparison of values for $W_{c}$ is not pertinent.

$$
\begin{array}{llll}
W_{c} & \Lambda_{0} & \nu & y
\end{array}
$$

Present work $15.0(1) \quad 0.57(1) \quad 1.60(5)-2.8(5)$

Band centre $16.54(1) \quad 0.576(2) \quad 1.57(2) \quad-2.8(5)$
Note also that $\Lambda_{0}$ does not directly depend on the initial choice of fitting parameters; instead, it is obtained at the end of the procedure, so the likelihood of this being a biased result is smaller than e.g. in the case of $\nu$. Universal critical amplitudes play an important rele in the statistical mechanics of critical phenomena 180 , so the present numerical agreement must be regarded as strong evidence in favour of universality of the transition (at least along the upper, non-reentrant, portion of the mobility edge, including the band centre).

To our knowledge, the only existing calculation of exponents along the mobility edge is that of Ref. 9 for Gaussian disorder (see their Table IV); however, their estimates are far too scattered, and no clear conclusion can be drawn from them.

In Table If we summarize our estimates for critical quantities at the upper transition; for comparison, corresponding values at the band centre are quoted. Error bars for the present work's results are somewhat arbitrary, but certainly encompass all well-behaved scaling plots. The exact coincidence of the estimate for $y$ with that of Ref. 11 must be regarded as fortuitous. The overall conclusion is that both transitions are in the same universality class.

\section{LOWER (REENTRANT) TRANSITION}

In Figure 4 are displayed the raw data for scaled localization lengths near the lower transition, whose approximate location is again estimated from Ref. 8.

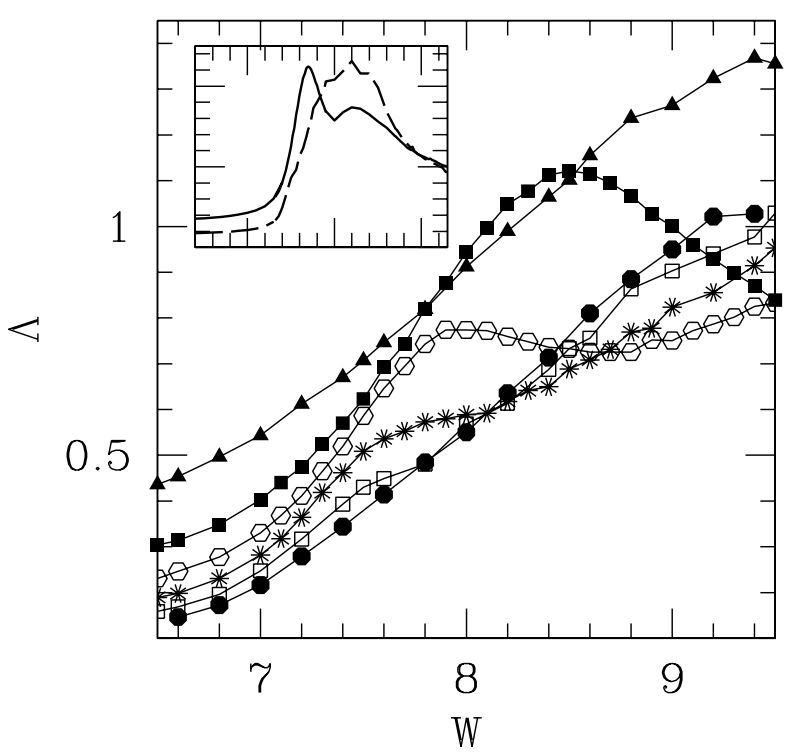

FIG. 4. Raw data for scaled localization lengths near the lower transition. Key to symbols is the same as in Fig. Z. Inset: raw data for $L=6$ (full line) and $L=12$ (dashed line), $2 \leq W \leq 16.5$. 
Data here behave rather differently from the upper transition. Non-monotonicity has been found earlier in this region; see data of Ref. 8 for $E=6.6,6.8, W=8$, $L=6-10$. A proper perspective is gained by looking at a broader range of $W$-variation, including both transitions: see the inset of Figure 4 , where only $L=6$ and 12 data are displayed, for clarity. The maxima shown in the main diagram are in fact shoulders, attributable to irrelevant scaling variables, much in the same way as the corrections discussed in Ref. 11 and Section III above. They must vanish for increasing $L$, and are distinct from the bona fide maxima, expected to be exhibited by finite-size data deep inside the conducting phase, somewhere between the two transitions. Indeed, the inset clearly shows that for $L=6$ the former effect is stronger than the latter, while for $L=12$ the trend has very much been reversed. For $L=4$ (not shown) shoulder and maximum (if any) merge, owing to finite-size broadening of the former.

The magnitude of distortions thus displayed makes it hard to account for them within a Taylor expansion framework, with a realistic number of parameters. Attempts to subtract the shoulders by postulating an ad hoc gaussian shape were not successful. We then decided to discard $L=4-8$ data, and try a fitting procedure similar to that given in Eq. (6) above, using only data for $L=10,12$, and 14 . Figure 5 shows the results of the best fit, with a set of parameters for which the (unweighted) $\chi^{2}=0.10$ for 61 data.

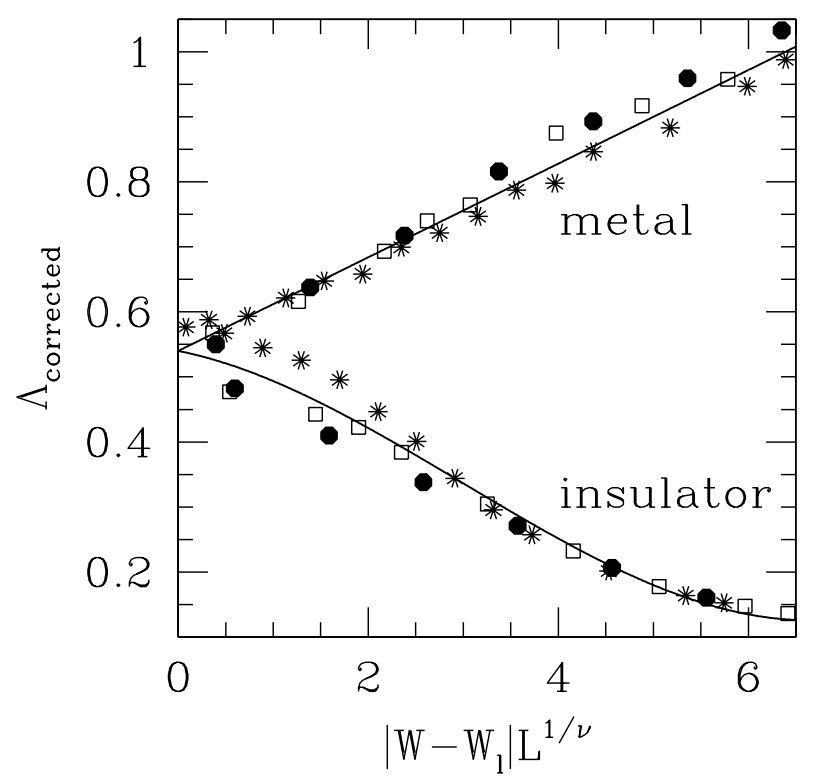

FIG. 5. Scaling plot near the lower transition. Here, $W_{l}=7.9, \nu=1.65, \psi=4.5, y=-3.2, a=-2.07, b=0$ (see Eq. (6)). Lines are guides to the eye, starting at $\Lambda_{0}=0.54$ on vertical axis. Key to symbols is the same as in Fig. 2; only data for $L=10-14$ are used.

One sees that the shoulder of $L=10$ data has been re- duced, but not totally eliminated; also, the overall quality of data collapse is inferior to, and the amplitudes of corrections larger than, the corresponding ones at the upper transition. However, the values $\nu=1.65$ and $\Lambda_{0}=0.54$ used in Fig. 5 are within $6 \%$ of those at the band centre. Bearing in mind the numerous assumptions made along the way, assigning error bars to these numbers would be somewhat risky. We have found that plots using $\nu$ just about anywhere in the $1.4-1.7$ range do not seem obviously much worse than the one displayed above; as regards $\Lambda_{0}$, anything between 0.5 and 0.6 seems possible, provided that one keeps $W_{l}$ in the range $7.8-8.2$. Therefore, the proper conclusion is that our data are not inconsistent with the lower transition being in the same universality class as that at the band centre (and, presumably, along the upper portion of the mobility edge).

\section{CONCLUSIONS}

We have found strong evidence that the transition along the upper portion of the mobility edge is in the same universality class as that at the band centre. We quote $\nu=1.60(5)$ and $\Lambda_{0}=0.57(1)$, to be compared respectively with $\nu=1.57(2)$ and $\Lambda_{0}=0.576(2)$ of Ref. 11 .

Our results for the lower (reentrant) transition are more mixed, though they certainly are compatible with universality: we give $\nu=1.55(15)$ and $\Lambda_{0}=0.55(5)$, from a qualitative visual analysis of trial scaling plots. One likely source for this would be the significant presence of finite-size corrections, linked to irrelevant scaling variables and/or nonlinearities in the scaling fields; so far, we have not been able to account for the bulk of such effects within a Taylor-expansion picture similar to that successfully employed, both at the band centre in Ref. 11 and at the upper transition in the present work.

Another, more fundamental, reason for such irregularities would be the failure of one-parameter scaling theory to describe the lower transition at all. It is argued in Ref. \& that "the critical behaviour should be independent of whether the energy $E$, the disorder $W$, or some combination of both, is taken as the scaling variable ... this is not generally true [e.g., in the present case] because the system shows two metal-nonmetal transitions when the disorder is changed for a fixed energy". It is also true that the physical mechanisms driving the lower transition (quantum tunnelling between tail states) and the upper one (quantum interference between essentially delocalized wave functions) are, in principle, distinct. Thus perhaps the one relevant parameter that represents the latter physical effect cannot do so for the former.

On the other hand, reasonably good scaling plots have been found here, by implicitly assuming the validity of one-parameter scaling, with corresponding numerical values of critical quantities which are not inconsistent with universality. This hints that the picture along both the upper and reentrant parts of the mobility edge may, in 
fact, be rather simple: critical behaviour would be dominated by a single fixed point, located at $E=0$.

More work, numerical and theoretical, is clearly necessary in order to settle the issue. It is also expected that experimentalists could be attracted into the discussion; metallic samples with suitably-located Fermi energies might conceivably be made to scan reentrant regions, by varying dopant concentrations.

\section{ACKNOWLEDGMENTS}

The author thanks Belita Koiller and J. A. Castro for interesting discussions, and Brazilian agencies CNPq (grant \# 30.1692/81.5), FAPERJ (grants \# E26171.447/97 and \# E26-151.869/2000) and FUJB-UFRJ for financial support.

${ }^{1}$ P. W. Anderson, Phys. Rev. 109, 1492 (1958).

${ }^{2}$ E. Abrahams, P. W. Anderson, D. C. Licciardelo, and T. V. Ramakrishnan, Phys. Rev. Lett. 42, 673 (1979)

${ }^{3}$ For a review of experiment and theory of localization, see e.g. A. MacKinnon and B. Kramer, Rep. Prog. Phys. 56, 1469 (1993).

${ }^{4}$ J.-L. Pichard and G. Sarma, J. Phys. C 14, L127 (1981); ibid. 14, L617 (1981).

${ }^{5}$ A. MacKinnon and B. Kramer, Phys. Rev. Lett. 47, 1546 (1981).

${ }^{6}$ A. MacKinnon and B. Kramer, Z. Phys. B 53, 1 (1983).

${ }^{7}$ B. Bulka, B. Kramer, and A. MacKinnon, Z. Phys. B 60, 13 (1985).

${ }^{8}$ B. Bulka, M. Schreiber, and B. Kramer, Z. Phys. B 66, 21 (1987).

9 T.-M. Chang, J. D. Bauer, and J. L. Skinner, J. Chem. Phys. 93, 8973 (1990).

${ }^{10}$ A. McKinnon, J. Phys.: Condens. Matter 6, 2511 (1994).

${ }^{11}$ K. Slevin and T. Ohtsuki, Phys. Rev. Lett. 82, 382 (1999).

12 T. Ohtsuki, K. Slevin, and T. Kawarabayashi, Ann. Phys. (Leipzig) 8, 655 (1999).

${ }^{13}$ K. Slevin, T. Ohtsuki, and T. Kawarabayashi, Phys. Rev. Lett. 84, 3915 (2000).

${ }^{14}$ H. Grussbach and M. Schreiber, Phys. Rev. B 51, 663 (1995).

${ }^{15}$ M. N. Barber, in Phase Transitions and Critical Phenomena, edited by C. Domb and J. L. Lebowitz (Academic, New York, 1983) Vol. 8.

${ }^{16}$ V. I. Oseledec, Trans. Moscow Math. Soc. 19, 197 (1968).

${ }^{17}$ A. Crisanti, G. Paladin, and A. Vulpiani, in Products of Random Matrices in Statistical Physics, Springer Series in Solid State Sciences Vol. 104, edited by Helmut K. Lotsch (Springer, Berlin, 1993).

18 J. L. Cardy, Scaling and Renormalization in Statistical Physics (Cambridge University Press, Cambridge, 1996), Chap. 3.
${ }^{19}$ W. Press, B. Flannery, S. Teukolsky, and W. Vetterling, Numerical Recipes in Fortran, The Art of Scientific Computing, 2nd ed. (Cambridge University Press, Cambridge, 1994), Section 15.5.

${ }^{20}$ V. Privman, P. C. Hohenberg, and A. Aharony, in Phase Transitions and Critical Phenomena, edited by C. Domb and J. L. Lebowitz (Academic, New York, 1991) Vol. 14. 